\title{
Real Time Physiological Status Monitorinig through Telemetry System for on-Spot-Accident Patients using IoT
}

\author{
S. Rabia Jebin ${ }^{1}$, N. Mohamedbeemubeen ${ }^{2}$ \\ ${ }^{1}$ Assistant Professor, 2 UG Student \\ 1,2Department of Electronics\& Communication Engineering, IFET College of Engineering Villupuram, India
}

\begin{abstract}
How to cite this paper: S. Rabia Jebin | N. Mohamedbeemubeen "Real Time Physiological Status Monitorinig through Telemetry System for on-SpotAccident Patients using IoT" Published in International Journal of Trend in Scientific Research and Development (ijtsrd), ISSN: 24566470, Volume-3 | Issue-3, April 2019, pp.1580-1583, URL: https://www.ijtsrd.c om/papers/ijtsrd23 470.pdf

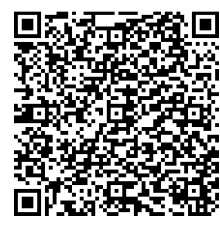
IITSRD23470
\end{abstract}

Copyright (C) 2019 by author(s) and International Journal of Trend in Scientific Research and Development Journal. This is an Open Access article distributed under the terms of the Creative Commons

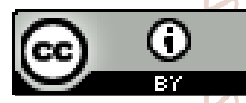
Attribution License (CC BY 4.0) (http://creativecommons.org/licenses/ by $/ 4.0$ )

\section{INTRODUCTION}

Internet of Things (IoT) is an ecosystem of connected physical objects and things that are accessible through the internet. The 'thing' in IoT could be a person with a heart as a monitor or an automobile with built-in-sensors, i.e. objects that have been assigned with an IP address and have the ability to collect and transfer data over a network without manual assistance or intervention. The embedded technology in the objects helps them to interact with internal or the external environment, which in turn affects the decisions taken.The Internet of Things is the network of physical devices, vehicles, home appliances, machines and other items embedded with electronics, software, sensors, actuators, and connectivity which enable these things to connect and exchange data, creating opportunities for more direct integration of the physical world into computer-based systems, resulting in efficiency improvement, economic benefits, and reduced human exertions.

IoT devices can enable remote health monitoring and emergency notification system. These health monitoring devices can range from blood pressure and heart rate monitors to advanced devices capable of monitoring $\mathrm{s}$ pecialized implants, such as pacemakers, fitbit electronic wristbands, or advanced hearing aids. Some hospitals have begun implementing "smart beds" that can detect when they are occupied and when a patients is attempting to get up. It can also adjust itself to ensure appropriate pressure and support is applied to the patient without the manual interaction of doctors and nurses.

Telemetry is a technology that allows remote measurement of signals and reporting of information. The word telemetry is derived from Greek wordstele(remote) and metron(measure). Telemetry is an automatic recording and transmission of data from remote or inaccessible sources to an IT system in a different location for monitoring and analysis. Telemetry data may be relayed using FM radio, infrared, ultrasonic, GSM, satellite or cable; depending on the application (telemetry is not only used in software development, but also in meteorology, intelligence, medicine, and other fields).Many modern telemetry systems take advantages of the low cost and ubiquity of GSM networks by using SMS to receive and transmit telemetry data. Bio telemetry is the measurement of biological parameters over long distance and for conveying biological information from a living organism and its environment to different locations, where this can be recorded. This involves radio frequency signals as a carrier for modulation, referred to as radio telemetry. 


\section{METHODOLOGY}

Telemetry is the sensing and measuring of information at some remote location and then transmitting that information to a central or host location. There, it can be monitored and used to control a process at the remote site in different location. The basic concept of the telemetry has been in existence for more centuries. Various methods of transmitting data or information from one site to another have been used. Telemetry using radio waves or wireless system offers several distinct advantages over other transmission methods. Some of these advantages are:

$>$ Faster output response time

$>$ No transmission lines to be cut or broken or damage

$>$ Lower cost compared to leased wire lines

$>$ Ease of use in remote areas where it is not practicalor possible to use wire or coaxial or optical cables

$>$ Easily relocate

$>$ Functioning over a wide range of operating and various environmental conditions.

\section{A. Wireless Telemetry System:}

Telemedicine has reduced the efforts of human by replacing wired infrastructure with wireless infrastructure. The signal transmission medium used here is a radio link, comprising a transmitting antenna (TA), a receiving antenna(RA) and the space between the two antennas are used for propagation of radio wave from TA to $R A$. The transmitter comprises a RF modulator (AM or FM type, depending on the performance, bandwidth, application and cost considerations) and an RFamplifier. The receiver consists an amplifier and a demodulator (AM or FM type as required to match the type of the modulator).

\section{B. Wireless Bio-signal System:}

In this study, a wireless bio-signal system design is used for health monitoring which integrates both the extracting and monitoring of thebio signal such as ECG, EEG and EMG. The developed integrating system is used for wireless monitoring of patient's biopotential changes of the heart, neuronal activity of the brain and muscles of the body. Dry electrodes, biosignal amplifier and filters are used for the development of this wireless system. The system also allows the continuous monitoring and graphical representation of the health condition of patient on a computer screen, even if the physician is away from the patient.

\section{Wireless Bio-Telemetry System Using FM Stereo Method:}

Development of telemetry system is used for Transmission and detection of vital body signals like temperature, heart sound etc. The transmitting unit detects and sends various kinds of patient's vital signals. The stationary receiving unit receives and analyzes the signals and alerts doctors and care takers about the health conditions of the patient if any necessary actions to be taken. This proposed system could be simplified and minimized in size by using commercialized electronic components. This system is to inform doctors and care takers about patient condition continuously. In these system two parameters namely temperature and heart rate are measured. If abnormalities in any one of the parameters are detected, it will be automatically informed to the doctor through FM receiver antenna. Temperature is sensed by using, two terminal IC AD590 temperature sensor which gives current output directly proportional to absolute temperature variations. This is amplified using instrumentation amplifier, and read the amplified output by using analog and digital converter. A microphone sensor is used to detect patient's heart beat sound. The standard FM stereo modulation method and a commercialized single IC chip were incorporated to reduce the size of the transmitter and to increase the signal-to-noise ratio. In the receiving unit a standard FM receiving demodulator module could be used to restore the original signal with very low cost. After detection of various signals, they can be processed by digital filters, and the extracted patients' parameters are displayed on CRT monitor.

\section{Comarch HTA Comprehensive Telemetry System For Hospital:}

Comarch HTA (Comarch Hospital Telemetry Assistant) is an innovative telemetry system developed with intensively hospitalized patients who require all time (24/7) monitoring in mind. The system allows continuous monitoring of a patient's health condition, as well as insight into their medical history, thus improving the efficiency of parental care through more effective time management. Key information, sent via android mobile devices worn by the patients, are available on stationary computers, as well as on tablets. This allows a quicker response time to any risks to a patient's life or health, which improves the quality of healthcare monitoring services.

\section{Functionalities:}

$>$ Parallel monitoring of the condition of a group of patients (up to 40 people).

$>$ Continuous registration of a patient's physiological signals (ECG, EEG, EMG, Heart beat and body temperature)

Automatic algorithms to detect irregularities in vital signs of patients.

Option for patients to call medical staff and their care takers.

$>$ Algorithm for detecting when a patient condition collapses.

$>$ It detects the Patients' location in the medical center.

$>$ Viewing the history of physiological records of the patients.

$>$ Pateints information is integrated with the hospital information system using IoT (for example ComarchOptimed NXT).

$>$ Automatic detection of irregularities in the operation of the utility network and telemedicine devices based on IoT.

\section{Examples of use:}

$>$ Cardiovascular diseases

$>$ Neurological diseases

$>$ Muscoskeletal disorders

$>$ Respiratory diseases

$>$ DiabetesPatients with cognitive disorders

$>$ (dementia)

$>$ Diagnostics

$>$ After surgical procedures

$>$ Multiple diseases

\section{E. Android Based Biomedical Signal Monitoring System:}

This proposed system is based on body area network (BAN) topology. All the sensors are attached to the human body and they are connected to microcontroller through BAN. Sensor measure the values of temperature, heart rate and this values are send to the Smart phones and computers having 
android application. Android application take the values compared it with threshold value that are already set in application using IoT, if abnormalities occur then the alert message will sends to the doctor and patients personal care taker. This represents low cost, web based system for transmission of various bio-signal, so that bio-signal acquired from the sensors can be transmitted from phone to the web server, so that doctor can analyses the signal of their patient properly even at outside the hospital.

\section{PROPOSED METHODOLOGY:}

The project object is to monitor the unconscious person's health status in real time and notify relevant doctor or nurse instantly without delaying for treatment if he/she is in risk. The complete project methodology is explained below in figure 1,

STEP 1 : Connecting all the electrodes and sensors to the patient and transferring that collected data through Wifi transmitter to the central nurse station.

STEP 2 : Once all the parameters are received by the PC based application through Wifi receiver, parameters are updated into database

STEP 3 : Android application fetches these parameters for every change and display on the smart phone application is shown in figure 2 .

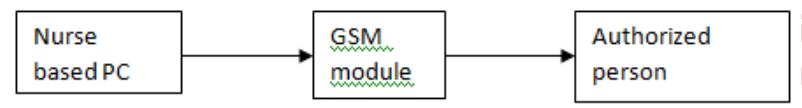

Figure 1: Notification for Authorized Person

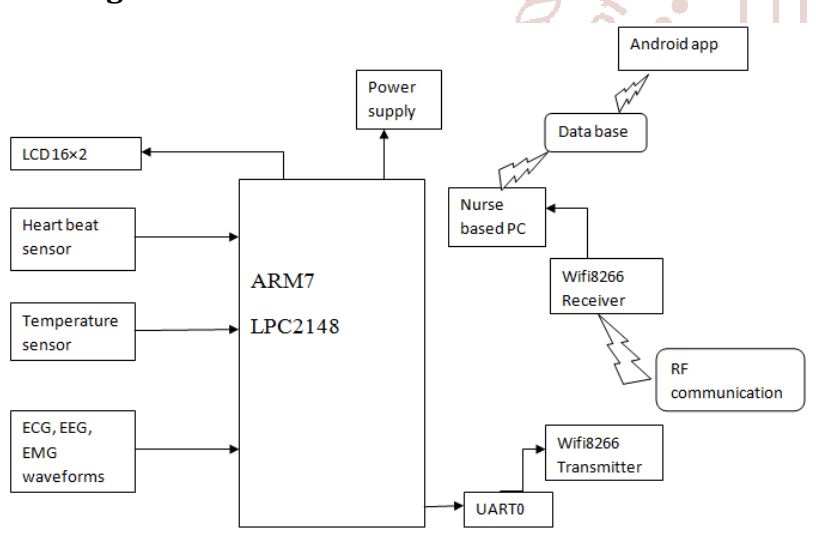

Figure 2: Architecture of the Navigation System

\section{DESCRIPTION:}

The general block diagram is shown in the figure 1. It consists of the following blocks

$>$ Temperature measuring part

$>$ Heart Sound-Detecting part

$>$ ECG,EEG \& EMG waveforms Measuring part

$>$ Telecommunications part

\section{A. HARDWARE TOOLS}

1. Temperature Measuring Part:

Temperature Sensor(LM35): The LM35 arrangement are accuracy incorporated circuit temperature sensors, whose yield voltage is linearly relative to the Celsius (Centigrade) temperature. The LM35's low impedance, linear output, and exact characteristic alignment make interfacing to readout or control hardware particularly simple, it has low selfwarming, under $0.1^{\circ} \mathrm{C}$ in still air.

\section{Heart Sound-Detecting Part}

Heartbeat Sensor: The new form utilizes the TCRT1000 intelligent optical sensor for photoplethysmography. It produces the digital output which is synchronous with the heartbeat. The output heartbeat can be nourished to either an ADC channel or an advanced info pin of a microcontroller for further preparing and recovering the heart rate in beats per minute (BPM).

\section{ECG,EEG \& EMG waveforms Measuring part}

Ag/AgCl Electrode: In this system very popular electrode silver/silver choloride ( $\mathrm{Ag} / \mathrm{AgCl})$ is used to sensing ECG, EEG and EMG signals. $\mathrm{Ag} / \mathrm{AgCl}$ electrodes allows current to pass across the interface between the electrolyte and the electrode which are nonpolarized electrodes. By this system, ECG can take from the electrode positioning on the chest, EMG from the muscle biceps and EEG from the scalp and forehead.

\section{Microprocessor Kit}

Power Supply: The power supplies are intended to change over high voltage $\mathrm{AC}$ mains power to a reasonable low voltage supply for electronic circuits and different gadgets. A power supply can be separated into a progression of obstructs, each of which performs a specific functionality. A DC power supply which keeps up the yield voltage steady regardless of AC mains changes or load varieties is known as "Controlled D.C Power Supply" For case a 5V directed power supply system.

ARM7 LPC2148 Microcontroller: The LPC2148 microcontroller depends on a 16/32-bit ARM7TDMI-S CPU that join the microcontroller with $32 \mathrm{~kb}, 64 \mathrm{~kb}, 128 \mathrm{~kb}, 256$ $\mathrm{kb}$ and $512 \mathrm{~kb}$ of flash memory of high speed. A128-bit wide memory interface and special architectures enables 32-bit code execution at most extreme clock rate.

Liquid-Crystal Display (LCD): Liquid crystal display is a level board show, electronic visual presentation that uses the light balance properties of liquid. Liquid crystals don't radiate light specifically. The parameters like temperature, ECG and BP is displayed.

\section{Telecommunications Part}

WIFI8266: Data uploading can be done using WIFI8266 module that may result faster uploading of data into the database and also enhancing the android application functionality.

GSM Module: SIM800H is a complete Quad-band GSM/GPRS solution in a LGA type which can be embedded in the customer applications. SIM800H support Quad-band $850 / 900 / 1800 / 1900 \mathrm{MHz}$, it can transmit voice, SMS and data information with low power.

\section{B. SOFTWARE TOOLS:}

KEIL IDE: Integrated development environment for embedded $C$ programming which enables us to code the microcontroller as per desired functionality. It is flexible and user friendly platform for programmers. The $\mu$ Vision Debugger gives a standalone environment in which testing, verification and optimization can be done on application code.

FLASH MAGIC: Flash Magic is an application created by Embedded Systems Academy to permit you to effortlessly get to the components of a microcontroller. With this program you can clear blocks or the whole flash memory of the microcontroller. 
VISUAL STUDIO: Visual Studio is an integrated development environment (IDE) developed by the Microsoft. It is utilized to create PC programs for Microsoft Windows, and in addition sites, web applications etc.

ECLIPSE IDE: The Eclipse Platform is an open source platform and toolbox for developing IDE's. Eclipse itself comprises of core innovations. A core Java Development Toolkit, and what is known as the Plug-in Development Kit. In this we are using eclipse to develop android application using java based android language.

\section{APPLICATIONS}

This project is particularly useful for the patients in the Intensive and Critical care unit wards, but it can also be extended to the general ward patients by using advance sensing devices. This method can be used for home care unit patients also by measuring the temperature level, if rising temperature is detected it can be said automatically to the doctor through transmitter. As same as body temperature, if there are abnormalities in heart beat rate it can be said automatically to the doctor who avoids the reverse flow of the blood from the body to bottle. This project can be used in large and multipurpose hospitals, where the doctor has to attend many critical case patients at a time. The developed system in this study can help those exercising patients vital signals can be monitored remotely in real time scenarios. Physical therapists can monitor patients' present quantity of exercise and decide the time and degree of physical therapy andcheck patient's vital safety.This enables the doctor to receive the current status of an accident patient in real time. The parameters of a particular patient go beyond a threshold value; an automated notification will pop up in doctor android mobile application. This android application can also generate call option if the doctor will not notify notification messages. Additionally system is also able to generate alarm to notify nurse around for emergency. treatment and by using GSM module the patient data's can also be send to the authorized person (i.e) friends, relatives etc. Ultimate goal of this project is to implement a low cost, high efficient and effective wireless real time system for health monitoring through telemetry system.

\section{CONCLUSION}

In the field of medicine and its monitoring system, IoT has a great significant role. IoT enables interoperability, machineto-machine communication, information exchange, and data movement that make healthcare service delivery effective. The health monitoring contributes a wide variety of applications such as hospital, home care unit, sports training and even in ambulance at traffic for emergency monitoring through telemetry system of the physiological status of injured persons in real time for taking immediate treatment without delaying. Obviously it is a solution to bridging the gap between the doctor and the patients and best to be used on rural areas. The health Monitoring system can wirelessly monitor the patient; notify the medical department and family members immediately in case of emergencies. Hence, this can truly give speedy administration for treatment and be advantageous in sparing a great deal of lives.

\section{REFERENCES}

[1] N.Surekha,N.Yamuna,A.J.Akil Kumar and K.G.Naveen Kumar, "Patient Monitoring System Using IoT," International Journal of Innovative Research in Advanced Engineering (IJIRAE),pp.177-182,Issue05,Vol-05,May 2018.

[2] C.Senthamilarasi, J.Jansi Rani, B.Vidhya and H.Aritha, "A Smart Patient Health Monitoring System Using IoT," International Journal of Pure and Applied Mathematics,pp.59-70,Vol.119,No.16, 2018.

[3] Yedukondalu Udara, Srinivasarao Udara, Harish H M and Hadimani H C, " Health Monitoring System Using IoT,"International Journal of Engineering and Manufacturing Science, pp. 177-182,Volume 8, Number 1 (2018).

[4] Ahmed Abdulkadir Ibrahim and Wang Zhuopeng, "Iot Patient Health Monitoring System," International Journal of Engineering Research and Application, , pp.77-80, Vol. 8, Issue 1, (Part -III) January 2018.

[5] M. A. Kumar and Y.R. Sekhar "Android Based Health Care Monitoring System," 2nd International Conference on Innovations in Information Embedded and Communication Systems, ICIIECS,IEEE,2016.

[6] S. M. Riazul islam, Daehan kwak, MD. Humaun kabir, Mahmud hossain, and Kyung-sup kwak," The Internet of Things for Health Care:A Comprehensive Survey" , DOI 10.1109/TDSC.2015.

[7] Home Based Health Monitoring System Using Android Smartphone,International Journal of Electical,Electronics and Data Communication, Vol-2, Issue-2, Feb-2014, Sushama Pawar, P.W.Kulkarni

[8] S. J. Jung and W. Y. Chung, "Flexible and scalable patient's health monitoring system in LoWPAN," Sensor Lett., vol. 9,no. 2, pp. 778-785, Apr. 2011.

[9] G. Y. Jeong,K. H. Yu, and Kim.N. G. Continuous blood pressure monitoring using pulse wave transit time.In International Conference on Control, Automation and Systems (ICCAS), 2005.

[10] K. Hung, Y. T. Zhang, and B. Tai. Wearable medical devices for telehome healthcare.In Procs.26th Annual International Conference on the IEEE EMBS, 2004. 